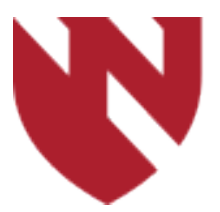

September 2020

\title{
Acute Colonic Ischemia Secondary to Oral Contraceptive Use
}

\author{
Lauren M. Keim \\ University of Nebraska Medical Center \\ Ian Cormier \\ University of Nebraska Medical Center \\ Brett Henderson \\ University of Nebraska Medical Center \\ Mark Mailliard \\ University of Nebraska Medical Center
}

Tell us how you used this information in this short survey.

Follow this and additional works at: https://digitalcommons.unmc.edu/gmerj

Part of the Higher Education Commons, and the Medicine and Health Sciences Commons

\section{Recommended Citation}

Keim, L. M., Cormier, I., Henderson, B., , Mailliard, M. Acute Colonic Ischemia Secondary to Oral Contraceptive Use. Graduate Medical Education Research Journal. 2020 Sep 29; 2(1).

https://digitalcommons.unmc.edu/gmerj/vol2/iss1/62

This Conference Proceeding is brought to you for free and open access by DigitalCommons@UNMC. It has been accepted for inclusion in Graduate Medical Education Research Journal by an authorized editor of DigitalCommons@UNMC. For more information, please contact digitalcommons@unmc.edu. 


\section{Acute Colonic Ischemia Secondary to Oral Contraceptive Use}

Creative Commons License

(c) (i)@(ङ)

This work is licensed under a Creative Commons Attribution-Noncommercial-No Derivative Works 4.0 License. 
anticoagulation. CT angiography confirmed the clot and revealed extensive compression of the proximal left common iliac vein by the right common iliac artery suggestive of May-Thurner syndrome. Intravenous heparin anticoagulation was initiated and apixaban held, but there was suspicion for development of heparin induced thrombocytopenia so he was transitioned to argatroban for anticoagulation.

Methods: Physical Exam, Doppler Ultrasound, CT Angiography
Conclusion: To manage May-Thurner syndrome he underwent manual thrombectomy with endovascular stent placement. The following night he developed worsening pain and swelling in his left leg consistent with compartment syndrome. He underwent four compartment fasciotomy and was restarted on argatroban. Repeat Doppler ultrasound revealed recurrence of extensive left lower extremity DVT. Given multiple recurrent DVTs despite adequate anticoagulation, dedicated peripheral blood smear was obtained and revealed atypical myeloblasts concerning for acute myeloid leukemia. Following confirmatory bone marrow biopsy, he was started on induction chemotherapy. He remained in hospital receiving ongoing chemotherapy after residual disease was detected on bone marrow biopsy following initial chemotherapy cycles.

https://doi.org/10.32873/unmc.dc.gmerj.2.1.059

\section{Vanishing Bile Duct Syndrome: A Paraneoplastic Phenomenon Secondary to Occult Hodgkin's Lymphoma \\ Lauren M. Keim ${ }^{1}$, Ian Cormier}

${ }^{1}$ University of Nebraska Medical Center, Department of Internal Medicine

Mentor: Marco Olivera-Martinez

Program: Internal Medicine

Type: Case Report

Background: A 27-year-old female with no medical history presented with one month of progressively worsening jaundice and a 30-pound weight loss. She was found to have conjugated hyperbilirubinemia with an acute cholestatic hepatitis. Ultrasound of the liver was unremarkable and magnetic resonance cholangiopancreatography revealed normal appearing intrahepatic and extrahepatic bile ducts without mechanical obstruction. An extensive workup for autoimmune and infectious etiologies of hepatitis was unremarkable. A liver biopsy was significant for features of cholestasis and ductopenia suggestive of vanishing bile duct syndrome (VBDS). A computed tomography scan of the chest revealed a large complex anterior mediastinal mass with extensive lymphadenopathy (Figure 1). A core needle biopsy of the right supraclavicular lymph node was obtained, and pathology was consistent with Hodgkin's lymphoma. She was initiated on induction therapy with improvement in her liver enzymes.

Methods: Ultrasound, CT scan

Conclusion: VBDS refers to a group of disorders with variable etiologies that cause a progressive destruction of intrahepatic bile ducts with resulting cholestatic liver injury. The diagnosis is made pathologically and requires a paucity of interlobular bile ducts, defined as less than $50 \%$ of portal areas with a bile duct on biopsy. Etiologies include infections, ischemia, autoimmune conditions, drug reactions, and malignancy. Treatment depends on the underlying cause and includes withdrawal of offending medications, immunosuppression, ursodeoxycholic acid, and treatment of malignancy in patients with paraneoplastic VBDS. Those with irreversible hepatic failure should be considered for liver transplantation. Prognosis is variable as some progress to cirrhosis and others have regeneration of biliary epithelium with recovery of hepatic function.

https://doi.org/10.32873/unmc.dc.gmerj.2.1.061

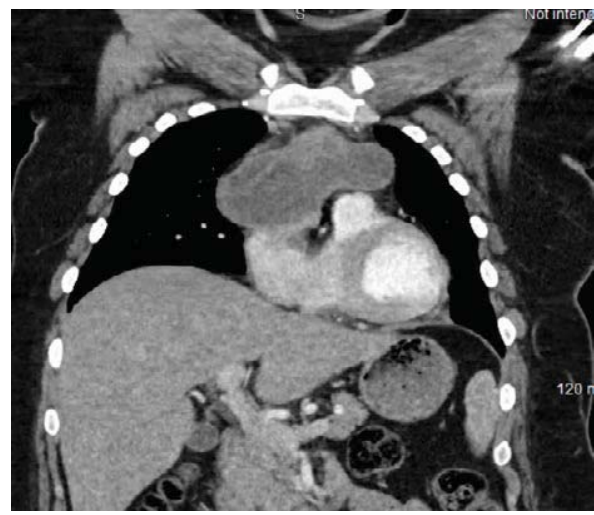

Figure 1. Computed tomography scan of the chest demonstrating large anterior mediastinal mass.

\section{Acute Colonic Ischemia Secondary to Oral Contraceptive Use}

Lauren M. Keim ${ }^{1}$, Ian Cormier ${ }^{1}$, Brett Henderson ${ }^{2}$, Mark Mailliard ${ }^{2}$

${ }^{1}$ University of Nebraska Medical Center, Department of Internal Medicine

${ }^{2}$ University of Nebraska Medical Center, Department of Internal Medicine, Division of Gastroenterology

\section{Mentor: Mark Mailliard}

Program: Internal Medicine

Type: Case Report

Background: A 39-year-old female presented with sudden onset of abdominal pain and hematochezia. Her medical history was significant for obesity and hypertension. The abdomen was tender to palpation in the left lower quadrant without rigidity. Workup was significant for leukocytosis and elevated inflammatory markers. Her stool was negative for gastrointestinal pathogens, including
Clostridium difficile. A colonoscopy was performed and revealed a diffusely edematous, ulcerated and violaceous segment of the sigmoid colon concerning for severe ischemic injury (Figure 1). Biopsies of the mucosa were obtained, and pathology confirmed ischemic colitis. Review of medications was significant for an oral contraceptive which was discontinued due to suspicion for drug induced colonic ischemia. The patient's hematochezia and abdominal pain resolved with conservative treatment.

Methods: Physical Exam, Colonoscopy
Conclusion: When young adults present with hematochezia, infectious colitis, inflammatory bowel disease, and hemorrhoids are commonly considered. However, intestinal ischemia is an important differential diagnosis. Ischemic colitis is the most common form of intestinal ischemia, generally encountered in patients greater than 50 years old as a result of nonocclusive hypoperfusion. Rarely, ischemic colitis may result from drug exposure. Mesenteric thrombotic events linked to contraceptive medications have been described in the literature and are thought to be secondary to a hypercoagulable 
state. Several medications, including hormonal therapies, constipation inducing drugs, laxatives, and illicit drugs have been associated with ischemic colitis. Taking a thorough medication history is essential when a patient is diagnosed with ischemic colitis. The recognition of medication induced colonic ischemia and prompt

discontinuation of offending agent is vital to patient outcomes.

https://doi.org/10.32873/unmc.dc.gmerj.2.1.060

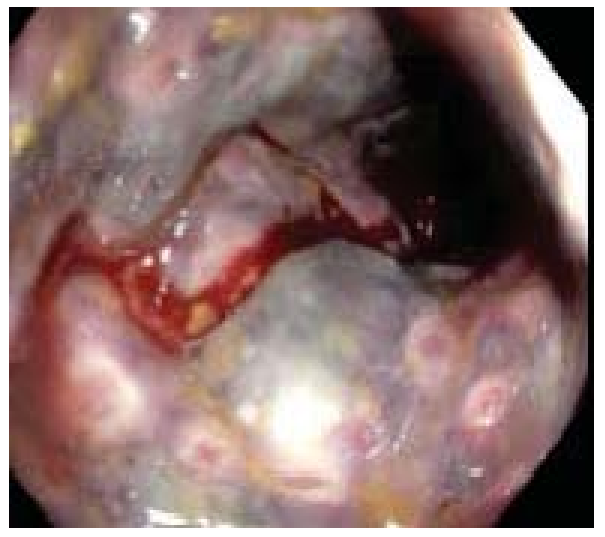

Figure 1. Colonoscopy demonstrating a diffusely edematous, ulcerated, and violaceous segment of the sigmoid colon

\section{Rural Otolaryngology - A Review of Resident Education and the Impact on Future Practice Selection Lauren Klute', Christie Barnes', Matthew Johnson 1,2, Kari Nelson ${ }^{3}$ \\ ${ }^{1}$ University of Nebraska Medical Center, Department of Otolaryngology \\ ${ }^{2}$ Ear, Nose and Throat Physicians of Kearney, Kearney, NE \\ ${ }^{3}$ University of Nebraska Medical Center, Department of Graduate Medical Education}

\section{Mentor: Christie Barnes}

Program: Otolaryngology

Type: Original Research

Background: The University of Nebraska Medical Center, Department of Otolaryngology - Head and Neck Surgery (OHNS) established a rural residency rotation in Kearney, Nebraska, in 2016. Each resident spends two months on the service as a junior (years two or three), and two months as a senior (years four or five). It has been cited that residency rotations in rural practices are a significant factor in retention of family practice and surgical physicians, however, there is not similar data available for Otolaryngology. This study provides data from current and prior OHNS residents on their rural rotation experience and the factors that contributed to future practice decisions.

Methods: In this qualitative study, we used a semi-structured interview guide to probe rural rotation participants on their experience and the influence the rotation had on their future practice decisions.

Results: The rural rotation was influential in residents' selection of future practice and did encourage residents to pursue rural practice. The benefits of the rotation included increased autonomy, diversity of cases, education in the private practice environment, and improved quality of life. The disadvantages included time away from family.

Conclusion: The University of Nebraska Medical Center OHNS Rural Rotation provides invaluable experience, education, and training. The rotation was influential in the selection of future practices and encouraged several residents to pursue rural Otolaryngology practices. A rural rotation experience in residency may lead to retention of Otolaryngologists for rural areas.

https://doi.org/10.32873/unmc.dc.gmerj.2.1.062

\section{Problem and Creating Guidelines Matthew A. Mormino' \\ ${ }^{1}$ University of Nebraska Medical Center, Department of Orthopaedic Surgery and Rehabilitation \\ 2University of Nebraska Medical Center, College of Medicine \\ ${ }^{3}$ Loma Linda University Medical Center \\ ${ }^{4}$ University of Nebraska Medical Center, College of Public Health, Department of Biostatistics}

Patterns of Opiate Use and Prescription Practices in Isolated Orthopedic Trauma Part One: Defining the

David J. Kusin'1, Emily A. Boes², Zachary C. Bailey ${ }^{1}$, Erin L. Stockwell', Ryan E. Miller ${ }^{3}$, Kaeli K. Samson ${ }^{4}$, Sara M. Putnam¹, Justin C. Siebler ${ }^{1}$,

Mentor: Matthew A Mormino

Program: Orthopaedic Surgery and Rehabilitation

Type: Original Research

Background: About $80 \%$ of the global supply of opiates is consumed in the United States, yet opioid use is associated with significant morbidity and safer approaches to pain control exist1-15. The purpose of this two- part study is to quantify our opioid prescribing practices and to formulate guidelines for safe and effective opioid stewardship.

Methods: The quantity of opioids prescribed (as Morphine Milligram Equivalents) at discharge and in the 90 days after surgery in adults with operatively treated, isolated fractures was correlated to patient demographics, comorbidities, fracture characteristics, and patient reported pain control.
Results: There were 56 males (47\%) and 63 females $(53 \%)$. Ankle fractures were the most common injury (34.2\%). 9 (7.6\%) fractures were open. The mean VAS pain scores at the first and second postoperative visits were 4.1 and 3.4, respectively. The mean initial quantity of opiates prescribed for all patients was $390 \mathrm{MME}$ and the mean total quantity was 535 MME (range 60-1800, Stdev 256; 60-2550, Stdev 245, respectively). 44\% of patients were prescribed refills. Greater 